\section{El estado del sector retail bajo una crisis sanitaria global}

The state of the retail sector under a global health crisis

\section{Mauricio Paredes Sandoval*}

\section{RESUMEN}

El advenimiento de una crisis sanitaria a nivel global provocó cambios drásticos en la vida de las personas, en todo orden: vida en el hogar, trabajo, estudios, vacaciones, entretenimiento. La compra de bienes de consumo, obviamente, fue afectada también por la pandemia; sin embargo, tal afectación no la sufrieron en la misma proporción $\mathrm{ni}$ todas las personas $\mathrm{ni}$ todos los establecimientos de venta al detalle, o retail. Este trabajo hace énfasis, precisamente, en las distintas formas en que tanto consumidores como negocios del retail han sentido esos cambios, los retos a los que se han visto enfrentados, las decisiones que han tomado, y los caminos que podrán escoger en el futuro cercano: los primeros para satisfacer sus necesidades y los segundos para ganarse el favor de los clientes y crear una oferta consistente de productos y servicios adecuados a la realidad en época de crisis.

Palabras clave: Pandemia, sector detallista, compradores, estrategias, tendencias, canales de venta.

\footnotetext{
* Ingeniero Comercial, Coordinador de Marketing, Instituto Edwards Deming, Quito Ecuador, m.paredes@deming.edu.ec, https://orcid.org/0000-0001-9785-4579
}

\section{REVISTATECNOLÓGICA ciencia y educación Edwards Deming}

ISSN: 2600-5867

\section{Atribución/Reconocimiento-NoCo} mercial- Compartirlgual 4.0 Licencia Pública Internacional - CC

\section{BY-NC-SA 4.0}

https://creativecommons.org/licenses /by-nc- sa/4.0/legalcode.es

Editado por: Tecnológico Superior Corporativo Edwards Deming

Enero - Julio Vol. 5 - I - 202 I

https://revista-edwardsdeming.com/index.php/es e-ISSN: 2576-097I

Recibido: 04 Marzo 2020

Aprobado: II Diciembre, 2020

Pag 98- 108 


\section{ABSTRACT}

The advent of a global health crisis caused drastic changes in people's lives, in every aspect: life at home, work, studies, vacations, entertainment. The purchase of consumer goods, obviously, was also affected by the pandemic; however, this impact did not affect all individuals or all retail establishments in the same proportion. This work precisely emphasizes the different ways in which both consumers and retail businesses have felt these changes, the challenges they have faced, the decisions they have made, and the paths they may choose in the near future: the former to satisfy their needs, and the latter to curry favor of customers and create a consistent offer of products and services in line with reality in times of crisis.

Keywords: Pandemic, retail sector, shoppers, strategies, tendencies, trading channels.

\section{INTRODUCCIÓN}

El 3 I de diciembre de 2019, la Comisión Municipal de Salud de Wuhan (China), reportó un grupo de casos de neumonía. Eventualmente se identificaría como un nuevo coronavirus.

El 29 de febrero se declaró el ingreso al Ecuador del primer infectado con coronavirus SARS-CoV-2.

El II de marzo de 2020, preocupada por los niveles de contagio y la severidad de la enfermedad, la Organización Mundial de la Salud caracterizó a la enfermedad del COVID-19 como pandemia.

El 16 de marzo de 2020, el presidente del Ecuador, declaró el Estado de Excepción, con el propósito de contener la transmisión del coronavirus.

Estos hitos en la cronología de la pandemia del coronavirus, marcan el inicio de un cambio radical, a escala mundial, de las relaciones entre personas y negocios, y una nueva forma de convivencia.

El modelo de negocio retail (o al detalle) se caracteriza por la venta de bienes de consumo al cliente final (no para la reventa). De hecho, el retail es el sector con el que la mayoría de consumidores finales tiene relación comercial.

Sean el supermercado, el micromercado, la pequeña tienda de la esquina, la panadería, la farmacia, el restaurante de comida rápida o la tienda de prendas de vestir, el comercio retail es donde los consumidores satisfacen la compra de bienes de consumo inmediato, y lo hacen, en condiciones normales, mediante transacciones de bajo volumen y bajo valor relativos y con una frecuencia relativamente alta. No es una sorpresa que las relaciones entre consumidores y comercios del sector retail se hayan visto también profundamente alteradas por la irrupción la pandemia y esas condiciones normales hayan dado paso a otras diferentes, con distintos volúmenes, montos y frecuencias.

Las democracias liberales occidentales -con sus obvias diferencias entre los países que permiten la economía de mercado y el libre tránsito de bienes, personas y capitales- han visto afectado ese estado de "laissez-faire" (aunque imperfecto), propuesto por el 
liberalismo clásico, donde las transacciones entre privados están libres o casi libres de intervenciones y regulaciones.

Todos los países del mundo han adoptado -con distintos matices y grados, y con distintos resultados- muchas medidas, normas y reglas, con el fin de reducir el contagio del virus y sus consecuencias en la salud humana, pero, al mismo tiempo, tratando de afectar lo menos posible a la actividad comercial de sus habitantes.

El COE Nacional (mecanismo, del Sistema Nacional Descentralizado de Gestión de Riesgos, responsable de promover, planear y mantener la coordinación y operación conjunta en emergencias o desastres con los diferentes actores a nivel nacional), ha sido el actor principal encargado de la adopción y control de las medidas de contención del virus en el Ecuador; $y$, aunque en menor grado, lo han sido también los comités provinciales y municipales, quienes en una etapa más avanzada de la pandemia, asimismo han tenido un papel protagónico en la determinación de tales medidas.

Los negocios del sector retail han tenido que adecuar a esa nueva realidad y a esas medidas restrictivas: sus días y horarios de atención a clientes; la estructura del personal a su cargo; la adopción de medidas de bioseguridad preventivas para empleados y clientes; los horarios, frecuencia y volúmenes de transporte y bodegaje para sus proveedores y puntos de venta, lo que implica un obligatorio cambio al alza en su estructura de costos.

Incluso, no es que sólo hayan tenido que realizar cambios sobre un sistema de negocio ya probado, muchos han tenido que cambiar, o al menos adecuar, sus métodos y procedimientos para cumplir con las limitaciones, impuestas desde fuera por las autoridades o por la realidad del mercado en situación de pandemia, y autoimpuestas hacia adentro por su necesidad de supervivencia.

\section{MATERIALES Y MÉTODOS}

Ya hemos dicho que la crisis global del covid-19 afectó a la población en todo orden social, y la investigación académica no es la excepción. Es por esta razón que, sujetos todavía a restricciones sanitarias y riesgos de contagio, se ha optado por la investigación on-line, por un lado, y por la participación de profesionales en el ramo por otra, mediante la entrevista en profundidad y la revisión de informes.

Con la misma rapidez con que los mercados han respondido a los cambios del entorno, lo han hecho los medios especializados y las empresas consultoras y de investigación del ramo; $y$, con ello, la publicación on-line y en tiempo real de la información y la data tan necesaria para la toma de decisiones comerciales, especialmente en época de crisis.

La información de mercado no puede esperar los tiempos que el canon editorial estipula. En un par de meses o hasta pocas semanas esos datos pasan a ser una serie histórica más que una herramienta al uso. El libro, con su innegable utilidad para la formación académica, se constituye en este caso como una instrumento demasiado estático y lento. Sin embrago, mucha de la información requerida sólo está disponible vía investigaciones ad hoc, y con costos muy elevados, inalcanzables para los medios disponibles para este 
trabajo. Es por eso que parte de los hallazgos de esta investigación fue recabada gracias a la colaboración de dos expertos del medio -uno es parte de una marca internacional, el otro de uno de los retailers más grandes del Ecuador-, con amplia trayectoria en el mundo del retail, y con base en informes confidenciales de dos consultoras en investigación de mercados de renombre mundial, bajo condición de anonimato.

Por la naturaleza reservada de esa información, no se hace referencia a los nombres ni de los profesionales, ni de las empresas a las que representan, ni de las consultoras mencionadas, como tampoco se hace referencia a resultados específicos de esos informes que puedan derivar en su identificación. Por esta razón, este artículo se lo debe caracterizar como de opinión y tendencia general.

\section{RESULTADOS}

El año 2020 nos obligó a cambiar, con la fuerza de una crisis sanitaria global, nuestro sistema social y vital. El trabajo, el estudio, la interacción social ya no volverán a ser los mismos.

El retail es de los pocos sectores de la economía que pudieron permanecer operando, a pesar de las restricciones, algunos incluso tuvieron que ampliar sus horas de atención al público para poder satisfacer el incremento de la demanda en algunos canales.

La aparición de nuevas cepas del virus hace que, a pesar de las campañas de vacunación masiva, el peligro de contagios siga latente. Si a eso le sumamos otras variables como la realidad del estado de la economía o el nivel de desempleo, el reto para el retail sigue siendo enorme aún después de más de un año en pandemia.

Como en cualquier periodo de crisis, los gastos que primero se reducen son los de entretenimiento, viajes, diversión, entre otros superfluos, y ese gasto de lo destina a lo más esencial, buscando siempre las oportunidades para ahorrar.

Como la principal política para control de la pandemia, por parte del gobierno, fue el confinamiento total, y aunque posteriormente se moderaron esas medidas, todavía persisten ciertas restricciones a la movilidad. Eso ha permitido que sea el hogar el centro vital de muchos ecuatorianos: teletrabajo, estudios y compra on-line son sólo parte de las actividades que ahora se realizan en casa. Esto ha permitido el crecimiento acelerado a categorías de productos de consumo hogar. $Y$ se espera que esa importancia inusitada que ha recobrado el hogar se mantenga hasta regresar a una normalidad prepandemia. Dependiendo de cómo vaya evolucionando la coyuntura, el comprador va estar evaluando continuamente sus necesidades inmediatas, según su capacidad de compra, y escogerá la canasta y el canal que la situación del momento lo permita.

El retailer y las marcas, pensando en la importancia de la actividad en casa, deberá pensar en estrategias para seducir al consumidor por esa vía:

Potenciar el beneficio emocional del uso del producto, convertir al producto en parte de la vida activa en el hogar y no sólo como un satisfactor inmediato de una necesidad y trasladar puertas adentro el entusiasmo del producto que se consumía afuera. 
En este contexto, tienen ventaja las marcas que puedan posicionar sus productos como ideales para el uso en el hogar y que permitan al usuario o consumidor suplir los que utilizaba fuera de su casa.

Dentro de la competencia de los distintos canales en período de pandemia, es el canal tradicional y la venta por catálogo los mayores perjudicados. El autoservicio, en cambio se consolida como el beneficiario de la coyuntura después del primer remesón. Estos continúan creciendo, tanto en volumen como en valor.

Los supermercados, que por su área de atención mayor en promedio que la de un canal tradicional tipo, garantizan una menor exposición al contagio es el autoservicio más elegido por el cliente cauto.

Desde el inicio de la pandemia hasta la actualidad el consumo hogar se mantiene sostenidamente, mostrando definitivamente una inclinación hacia mayores volúmenes, formatos más grandes y menor frecuencia de compra.

A manera de comparación, estos datos (a marzo de 2021) revelan el comportamiento de ambos modelos en el Ecuador:

Discounter: Ticket medio $\$ 9.99$, frecuencia 40 días, unidades por acto II

Supermercados: Ticket medio $\$ 26.40$, frecuencia, II días, unidades por acto 17

El autoservicio les ha quitado protagonismo a los canales tradicionales. Como el comprador alarga los períodos de tiempo entre su aprovisionamiento, éste trata de adquirir toda su canasta en el mismo punto de venta, haciendo más significativa su compra.

Los supermercados que han desarrollado marcas propias han visto un crecimiento sobresaliente en ese rubro. El consumidor está demostrando lealtad con esas marcas.

Comparando el primer trimestre de este año, con el primer trimestre de 2020, el consumo del nivel socioeconómico bajo, a pesar de que no crece, sigue reportando el mayor peso poblacional, aun teniendo en cuenta que este target suele migrar a otros canales de descuento. Ver tabla a continuación:

\begin{tabular}{llllll}
\hline $\begin{array}{l}\text { Peso } \\
\text { poblacional }\end{array}$ & $\begin{array}{l}\text { Variación } \\
\text { valor }\end{array}$ & NSE & Ticket & Frecuencia & $\begin{array}{l}\text { Unidades por } \\
\text { acto }\end{array}$ \\
\hline $66 \%$ & $--\%$ & Bajo & $\$ 18.62$ & 13 días & 13 unidades \\
$27 \%$ & $+14 \%$ & Medio & $\$ 29.55$ & 9 días & 18 unidades \\
$7 \%$ & $+8 \%$ & Alto & $\% 51.02$ & 8 días & 28 unidades \\
\hline
\end{tabular}

El primer trimestre de 202 I ha resultado en el crecimiento del 75\% de las categorías, concentrándose especialmente en las categorías de consumo hogar.

El sector retail está en constante evolución, y la pandemia aceleró el cambio hacia las nuevas tecnologías y la responsabilidad social corporativa. Sin embargo, a medida que el sector y los consumidores vayan regresando hacia una cuasi normalidad, un hecho se mantiene constante: el precio sigue siendo el factor decisivo.

En este estado de cosas, los consumidores califican al precio asequible como el más alto de los valores clave, seguido de otros, tales como: la salud personal, el bienestar familiar y asuntos ambientales. 
La pérdida de empleos, la disminución de salarios, la quiebra de negocios, y en general toda la afectación socioeconómica, impulsan todavía más al precio como el factor de decisión de compra más importante. El comprador, incluso de clases más pudientes, ahora compara precios y hasta cambia de marca en algunas categorías de producto.

Como con toda crisis de grandes dimensiones la demanda sufre muchos cambios ya que el futro, aún a corto plazo, es muy incierto. El consumidor común busca ahorrar y por ende reduce sus compras sólo a lo indispensable. La política de precios de marcas y autoservicios debe tomar muy en cuenta la sensibilidad a los precios de cada uno de sus segmentos si quiere evitar que el comprador abandone el canal.

\section{Restricciones al retail por el coronavirus}

De la noche a la mañana tuvimos que alterar en gran medida las formas de cómo los consumidores compramos, ahorramos y gastamos. Incluso en temas tan habituales como los horarios de atención al público que estuvieron sujetos a las restricciones de la autoridad pública

El pequeño negocio de retail no tiene capacidad para implementar el teletrabajo, el servicio a domicilio y el cumplimiento estricto de medidas de bioseguridad y aforos permitidos. Todos estos asuntos implican procedimientos, costos y personal para cumplirlos y hacerlos cumplir.

El comercio retail también está derivando a otras formas de pago sin contacto (Contactless payment). Las tarjetas de crédito contactless y las aplicaciones móviles de pago o billetera virtual, evitan, precisamente, el contacto físico que tratamos de evitar por los contagios, sin embargo, no están al alcance de todos los compradores, y algunos de estos se resisten a adoptar todavía estas tecnologías a las que ven como poco seguras y propensas a fraude.

Los empleados del sector retail también están sujetos, por supuesto, a restricciones de pandemia y al cumplimiento de protocolos de bioseguridad. Es imposible tratar todas y cada una de estas, pero valga señalar algunas: distanciamiento social entre compañeros de labores y con clientes, transporte de personal y mercancías, limpieza y desinfección de superficies y lugares de trabajo, higiene personal y formas de saludar, ventilación áreas, etc.

Por su parte, los clientes también deben cumplir con normas de distanciamiento social. Medidas de bioseguridad al ingreso y dentro del establecimiento.

El cierre de fronteras, los toques de queda $y$, en general todas las restricciones de movilidad vehicular y de personas, complica el transporte de mercancías a toda la cadena de distribución y a la provisión en tiempo, lugar y condiciones que el retailer necesita para el servicio adecuado al consumidor final.

\section{Estado del consumidor}

La crisis sanitaria creó también una crisis económica. Por supuesto, no todos han sido afectados en la misma forma.

Hay consumidores que perdieron su trabajo, vieron sus ingresos reducidos notablemente o pasaron a la "economía informal". Estos no pueden honrar sus deudas y deben subsistir con lo más básico. 
Para otros consumidores el impacto no fue tan fuerte. Han podido mantener su trabajo, tienen los medios tecnológicos para hacer teletrabajo y lo que han hecho es trasladar su consumo desde fuera de casa hacia adentro.

La estrategia del retailer debe tener en cuenta estos perfiles tan distintos y entender las motivaciones de compra y las prioridades de unos y otros, ya que sus realidades implican una mezcla de mercadeo diferente para cada caso.

El consumidor actual ha pasado por una experiencia vital no esperada. Sus actitudes, expectativas, prioridades y comportamientos han sido reformadas por una crisis de proporciones globales. En condiciones prepandemia, los compradores realizaban desplazamientos más largos para llegar a su punto de venta favorito. O hacían un "mix" de visitas a distintos establecimientos que les presten las mejores condiciones en cada categoría de productos para ir llenando su canasta. Actualmente el comprador va al punto de venta que le quede más cerca, y compra en ese sitio toda su canasta.

EL nuevo comprador, ese miembro de la familia que se sacrifica por el resto para ir por los víveres, sabe lo que necesita comprar, pero sólo una vez frente al surtido de la percha toma la decisión final de compra.

Esta variación en la rutina de compras obliga al punto de venta a redefinir sus estrategias de mezcla de mercadeo, porque su perfil de usuario ha cambiado en época de crisis.

El "shopper" y el consumidor cada vez están más diferenciados. Las restricciones de aforo en los puntos de venta se han encargado de tener a un comprador cada vez más informado y a un consumidor más pasivo y alejado del acto propio de la compra.

El nuevo comprador, además, probablemente pertenece a otros segmentos de mercado a los que el establecimiento no apuntaba. Este nuevo shopper pertenecerá a otro NSE (nivel socio económico), tendrá otros comportamientos que los compradores habituales y sus estilos de vida y personalidad diferirá también de los de nuestros visitantes tradicionales.

El consumidor está demostrando que quieren que los puntos de venta sean algo más que un sitio para comprar (especialmente en los NSE más altos). El comprador actual apoya marcas y tiendas que se destacan por respaldar causas sociales como la protección del medioambiente, la discriminación, prácticas comerciales sustentables, la igualdad de género o la inequidad económica.

\section{E-commerce}

Entrega a domicilio, delivery, sólo para llevar, compra on-line, comercio electrónico, son términos normalizados en el vocabulario comercial desde aparición de la pandemia, sin embargo, los consumidores aún ansían tener experiencias de compra persona a persona. Muchos negocios que tuvieron desarrollos muy limitados en tecnología en años anteriores, han tenido que adaptarse en tiempo récord a un entorno digital para el que no estaban preparados.

La compra virtual ha salvado gran parte de las operaciones de muchos comercios, pero a medida que transcurre el tiempo los consumidores van regresando a sus canales de confianza. Es decir, el e-commerce se mantendrá, pero no para todos los segmentos. 
Quienes tuvieron una mala experiencia, o no conservadoramente no le tienen confianza al canal, no repetirán la experiencia a menos que sea estrictamente necesario. Otros, en cambio, que no confiaban en la compra digital y fueron atraídos por la comodidad y rapidez del delivery lo seguirán usando en desmedro del autoservicio.

Por eso, el esquema online debe ser mantenido y potenciado ya que la mayoría de consumidores, de aquí en más, irán saltando de un canal a otro: presencial y virtual, según el entorno y su experiencia lo promuevan.

Tan es así que el reto no está únicamente en contar con la plataforma tecnológica para la venta on-line, el desafío es hacer que la compra virtual sea una experiencia válida y placentera para el comprador, vía eficiencia, rapidez y seguridad.

El canal moderno fue el favorecido inicialmente por las medidas de restricción de movilidad, sus amplios portafolios garantizaron la provisión de muchos segmentos, sin embargo, la situación económica es un baño frío de realidad y muchos compradores fueron regresando a canales tradicionales más económicos y cercanos físicamente.

Las marcas necesitan dar un giro a una experiencia multicanal de calidad. Obviamente tienen que mantener la conveniencia de la compra on-line. El masivo incremento en el comercio electrónico y la reducción de tiempos en las entregas a domicilio son acciones comerciales que han logrado un gran desarrollo y no se detendrán con el fin de la pandemia. Pero mientras eso sucede también deben continuar proporcionando una experiencia "in-store" valiosa para los clientes que prefieren la experiencia personal en el punto de venta.

\section{El retail a futuro}

Los pronósticos y previsiones, trabajo complicado y hasta ingrato muchas veces, por la cantidad de variables en juego, se vuelve virtualmente una lotería en períodos de crisis. Pocas certezas y muchos factores imprevisibles empujan a las empresas a tomar decisiones de riesgo.

Si embargo, hay pautas de comportamiento de los mercados y sus consumidores que nos pueden dar pistas sobre lo que le espera al sector retail en un futuro cercano y las distintas estrategias que los comercios pueden pensar para su planificación operativa.

Si bien la primera etapa de la crisis nos obligó a enfocarnos en la sobrevivencia, la industria debe focalizar sus esfuerzos en el siguiente paso: la post-pandemia en el mediano plazo, y la nueva normalidad en el largo plazo

Los cambios en la economía mundial, la adopción de nuevas tecnologías, nuevas normas sociales y comportamiento diferente del consumidor, darán forma al retail del futuro.

La forma que tenga la curva de recuperación económica en cada mercado dependerá de la habilidad individual de cada país de controlar la pandemia.

La digitalización de sus procesos era una posibilidad, ahora es una necesidad.

A medida que las empresas entran en una era postpandemia las marcas tendrán que atraer a un consumidor que será muy diferente del que fue hasta el año 2020.

Las operaciones deben ser más flexibles y adaptables a una realidad con cambios cada vez más drásticos y rápidos. 
Habrá puestos de trabajo que podrán mantener, al menos parcialmente, el teletrabajo. La automatización y la robotización suplirán algunos puestos ocupados actualmente por personas.

Esa digitalización de las operaciones obliga al reforzamiento de los protocolos de ciberseguridad, tanto para le punto de venta como para el comprador, y que ambos se sientan seguros.

Las cadenas de distribución deberán también diseñarse a prueba de fallos para minimizar los "eslabones rotos" por restricciones a la circulación de mercancías, por un lado, y los abruptos cambios de demanda de determinadas categorías de producto, por otro.

Analizar el comportamiento del consumidor postpandemia se constituye como una tarea obligatoria para la redefinición de los portafolios de productos y servicios, y la apertura de nuevos canales de atención y comunicación interactivos.

Ese sentido de riesgo y peligro que aqueja al consumidor en la crisis sanitaria puede seguir acompañándolo incluso después del levantamiento de todas las restricciones. El retail tendrá que reinventarse para atender a ese cliente muy cauteloso que cambiará la manera en la que hace la compra: estará más pendiente de la higiene del punto de venta, sus visitas serán menos frecuentes pero sus compras más voluminosas y habrá categorías que prefiera comprarlas en línea.

El canal discounter, ya mejor desarrollado en otros países de la región, está ingresando con fuerza al Ecuador. Este modelo de canal, según la experiencia extranjera, afecta primero al canal tradicional y luego al canal moderno. Su "selling porposition" se puede resumir en: calidad a bajo precio.

El adaptador temprano de estos canales suele ser el consumidor habitual de marcas propias, y posteriormente es adoptado por personas de nivel adquisitivo más bajo, seducidos por la accesibilidad.

En toda Latinoamérica son los canales e-commerce y discounter los de mayor desarrollo. Esa experiencia presagia lo que puede suceder en el largo plazo con el comprador ecuatoriano, especialmente en discounter, que es un terreno bastante virgen todavía para el retail.

Ya hemos dicho que el consumo se dirigió especialmente al hogar. La mayor cantidad de ese gasto pertenece al rubro alimentos, seguido de aseo personal y limpieza del hogar; mientras categorías de productos menos esenciales como cosmética y OTC siguieron el camino contrario

Con respecto a las marcas en consumo masivo, apenas un crecimiento del $10 \%$ es atribuible a las marcas premium, el resto se lo llevan las marcas económicas y marcas propias.

Después de un año de pandemia ya se puede ver que hay cambios permanentes y no sólo reacciones pasajeras y circunstanciales

Con el proceso de vacunación en marcha y el paulatino levantamiento de las restricciones, los consumidores empiezan a intuir el regreso a sus hábitos normales de compra, aunque algunos creen que las prácticas de distanciamiento social serán muy difíciles de romper, a pesar de que ya no sean obligatorias. 
Ganarse la confianza de un cliente en condiciones normales ya es un reto que enfrenta cualquier negocio, ganarse la confianza en un esquema de pandemia global lo hace exponencialmente más difícil. El retail deberá generar una experiencia consistente en todas las interacciones y en todos los canales.

Las tiendas y marcas que hayan estado en posibilidad de adoptar canales de entregas a domicilio y venta online seguirán haciéndolo, pero deberán estar también preparadas para el regreso físico del comprador. Éste buscará, más allá de la compra del producto, el contacto social, la experiencia, en fin, un propósito más alto.

Hay que mirar al comprador en forma holística. No sólo cómo compra o paga, sino cómo vive y cómo trabaja. La gente no está sólo consumiendo más en su hogar, toda su actividad está ligada ahora a su sitio de vivienda: trabajo desde casa, menos viajes o salidas a comer y más cocina y entretenimiento en casa.

\section{DISCUSIÓN}

Incluso después de que la amenaza de la pandemia se haya superado, ni los competidores ni los retailers serán los mismos que antes, dando como resultado que las estrategias de mercado conservadoras no tendrán los mismos efectos en un entorno radicalmente diferente.

Si la competencia entre canales del retail es intensa en condiciones normales de un mercado libre, la competencia en época de crisis es feroz.

Los canales emergentes no reemplazarán por completo al canal tradicional ni moderno, pero la participación de los "nuevos chicos del barrio" se notará cada vez más.

Los compradores serán cada vez más multicanal y sólo los retailers que sepan estudiar las motivaciones de compra del nuevo comprador lograrán retenerlo.

En el mundo comercial postpandemia la "propuesta única de venta" ya no será tan "única". Marcas y retailers deben crear una promesa de valor diferente para cada canasta, para cada segmento y para cada canal.

\section{REFERENCIAS}

WHO Statement. (2020). Archived: WHO Timeline - COVID-19. Recuperado de https://www.who.int/news/item/27-04-2020-who-timeline---covid- 19

Redacción Ecuador Transparente. (2020). Se confirma coronavirus en Ecuador, 15 días después de haber ingresado. Recuperado de http://www.ecuadortransparente.com/noticias/pais/se_confirma_coronavirus_e n_ecuador_15_dias_despues_de_haber_ingresado.asp

Boletín Secretaría General de Comunicación de la Presidencia. (2020). El presidente Lenín Moreno decreta Estado de Excepción para evitar la propagación del COVID-19. Recuperado de https://www.comunicacion.gob.ec/el-presidentelenin-moreno-decreta-estado-de-excepcion-para-evitar-la-propagacion-delcovid-19/

Servicio Nacional de Gestión de Riesgos y Emergencias. (2018). Comité De Operaciones De Emergencia Nacional - Coe-N. Recuperado de 
https://manualcoe.gestionderiesgos.gob.ec/comite-de-operaciones-deemergencia-nacional-coe-n/

Forbes Staff. (2020). ¿Cómo ha cambiado la realidad del retail y consumo masivo en tiempos de contingencia mundial? Recuperado de https://forbes.co/2020/07/03/negocios/como-ha-cambiado-la-realidad-del-retaily-consumo-masivo-en-tiempos-de-contingencia-mundial/

Pulido, R. (2020). Estado del ecosistema retail: impacto del COVID-19 en el sector. Recuperado de https://www.geoblink.com/es/blog/estado-ecosistema-retailimpacto-covid-19/

Oyaga, L. (2020). Impacto del Covid-I 9 y Post Covid en el Retail Latinoamericano. Recuperado de https://clubdeltrade.com/blog/impacto-del-covid- I9-y-post-covid-en-el-retaillatinoamericano/

Díaz, F. et al. (2020). Guía post cuarentena para empleadores. Recuperado de https://storagemailing.blob.core.windows.net/archivos/CCQ\%20Gu\%C3\%ADa\% 20Post\%20Cuarentena\%20-\%20Empleadores.pdf

Graziani, E. (202I). 4 takeaways from the State of Retail and the Consumer. Recuperado de https://nrf.com/blog/4-takeaways-state-retail-and-consumer

Rogers, K. (2020). Future Consumer Index: How to serve the 'Anxious Consumer' after COVID19. Recuperado de https://www.ey.com/en_us/consumer-products-retail/howto-serve-the-anxious-consumer-after-covid- 19

Gramling, K. (2021). Future Consumer Index Cycle 6: How a year of pandemic changed consumers. Recuperado de https://www.ey.com/en_us/consumer-productsretail/future-consumer-index-cycle-6-how-a-year-of-pandemic-changedconsumers 